\title{
Face washing promotion for preventing active trachoma (Review)
}

\author{
Ejere HOD, Alhassan MB, Rabiu M
}

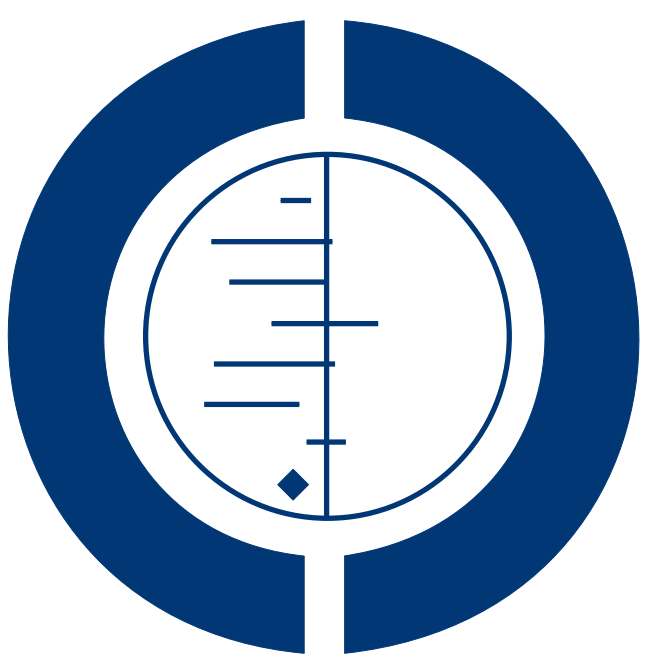

\section{THE COCHRANE COLLABORATION $^{\circledR}$}

This is a reprint of a Cochrane review, prepared and maintained by The Cochrane Collaboration and published in The Cochrane Library 2012, Issue 4

http://www.thecochranelibrary.com

\section{WILEY}


TABLE OF CONTENTS

HEADER . . . . . . . . . . . . . . . . . . . . . . . . . . . . . . . . . . . . 1

ABSTRACT . . . . . . . . . . . . . . . . . . . . . . . . . . . . . . . . . . . . . . . . . . . . . . .

PLAIN LANGUAGE SUMMARY . . . . . . . . . . . . . . . . . . . . . . . . . . . . . . . . . . . 2

BACKGROUND . . . . . . . . . . . . . . . . . . . . . . . . . . . . . . . . . . . . . . . . .

OBJECTIVES . . . . . . . . . . . . . . . . . . . . . . . . . . . . . . . . . . . . . .

METHODS . . . . . . . . . . . . . . . . . . . . . . . . . . . . . . . . . . . . . .

RESULTS . . . . . . . . . . . . . . . . . . . . . . . . . . . . . . . . . . . . . 5

DISCUSSION . . . . . . . . . . . . . . . . . . . . . . . . . . . . . . . . . . . . . 7

AUTHORS' CONCLUSIONS . . . . . . . . . . . . . . . . . . . . . . . . . . . . . . . . . 8

ACKNOWLEDGEMENTS . . . . . . . . . . . . . . . . . . . . . . . . . . . . . . . . . . . . . . . . . . .

REFERENCES . . . . . . . . . . . . . . . . . . . . . . . . . . . . . . . . . . . . . . 8

CHARACTERISTICS OF STUDIES . . . . . . . . . . . . . . . . . . . . . . . . . . . . . . . . . . . . .

DATA AND ANALYSES . . . . . . . . . . . . . . . . . . . . . . . . . . . . . . . . . . . . . . 12

ADDITIONAL TABLES . . . . . . . . . . . . . . . . . . . . . . . . . . . . . . . . . . 12

APPENDICES . . . . . . . . . . . . . . . . . . . . . . . . . . . . . . . . . . . . . . 12

WHAT'S NEW . . . . . . . . . . . . . . . . . . . . . . . . . . . . . . . . . . . . 15

HISTORY . . . . . . . . . . . . . . . . . . . . . . . . . . . . . . . . . . . . . . . . 15

CONTRIBUTIONS OF AUTHORS . . . . . . . . . . . . . . . . . . . . . . . . . . . . . . . . . .

DECLARATIONS OF INTEREST . . . . . . . . . . . . . . . . . . . . . . . . . . . . . . . . . . . . . . .

SOURCES OF SUPPORT . . . . . . . . . . . . . . . . . . . . . . . . . . . . . . . . . . . . . . . . . . . .

INDEX TERMS . . . . . . . . . . . . . . . . . . . . . . . . . . . . . . . . . . . . 16

Face washing promotion for preventing active trachoma (Review)

Copyright $\odot 2012$ The Cochrane Collaboration. Published by John Wiley \& Sons, Ltd. 


\title{
[Intervention Review]
}

\section{Face washing promotion for preventing active trachoma}

\author{
Henry OD Ejere ${ }^{1}$, Mahmoud B Alhassan ${ }^{2}$, Mansur Rabiu $^{3}$ \\ ${ }^{1}$ Phoebe Inpatient Medicine Specialists, Phoebe Putney Memorial Hospital, Albany, Georgia, USA. ${ }^{2}$ Clinical Ophthalmology, The \\ National Eye Centre, Kaduna, Nigeria. ${ }^{3}$ Prevention of Blindness Union, Riyadh, Saudi Arabia \\ Contact address: Henry OD Ejere, Phoebe Inpatient Medicine Specialists, Phoebe Putney Memorial Hospital, 425 W. Third Avenue, \\ Suite 500, Albany, Georgia, 31701, USA. hodejere2000@yahoo.com.
}

Editorial group: Cochrane Eyes and Vision Group.

Publication status and date: New search for studies and content updated (no change to conclusions), published in Issue 4, 2012.

Review content assessed as up-to-date: 2 September 2011.

Citation: Ejere HOD, Alhassan MB, Rabiu M. Face washing promotion for preventing active trachoma. Cochrane Database of Systematic Reviews 2012, Issue 4. Art. No.: CD003659. DOI: 10.1002/14651858.CD003659.pub3.

Copyright (C) 2012 The Cochrane Collaboration. Published by John Wiley \& Sons, Ltd.

\begin{abstract}
A B S T R A C T
Background

Trachoma remains a major cause of avoidable blindness among underprivileged populations in many developing countries. It is estimated that about 146 million people have active trachoma and nearly six million people are blind due to complications associated with repeat infections.
\end{abstract}

\section{Objectives}

The objective of this review was to assess the effects of face washing on the prevalence of active trachoma in endemic communities.

\section{Search methods}

We searched CENTRAL (which contains the Cochrane Eyes and Vision Group Trials Register) (The Cochrane Library 2011, Issue 8), MEDLINE (January 1950 to September 2011), EMBASE (January 1980 to September 2011), Latin American and Caribbean Health Sciences Literature Database (LILACS) (January 1982 to September 2011), the metaRegister of Controlled Trials ( $m$ RCT) ( www.controlled-trials.com) and ClinicalTrials.gov (www.clinicaltrials.gov). There were no date or language restrictions in the electronic searches for trials. The electronic databases were last searched on 2 September 2011. We checked the reference list of the included trials to identify further relevant trials. We used the Science Citation Index to search for references that cite the studies that are included in the review. We also contacted investigators and experts in the field to identify additional trials.

\section{Selection criteria}

We included randomized or quasi-randomized controlled trials, comparing face washing with no treatment or face washing combined with antibiotics against antibiotics alone. Participants in the trials were people normally resident in endemic trachoma communities.

\section{Data collection and analysis}

Two review authors independently extracted data and assessed trial quality. Study authors were contacted for additional information. Two clinically heterogeneous trials are included, therefore a meta-analysis was considered inappropriate.

Face washing promotion for preventing active trachoma (Review)

Copyright $\odot 2012$ The Cochrane Collaboration. Published by John Wiley \& Sons, Ltd. 


\section{Main results}

This review included two trials with data from a total of 2560 participants. Face washing combined with topical tetracycline was compared to topical tetracycline alone in three pairs of villages in one trial. The trial found a statistically significant effect for face washing combined with topical tetracycline in reducing 'severe' active trachoma compared to topical tetracycline alone. No statistically significant difference was observed between the intervention and control villages in reducing ('non-severe') active trachoma. The prevalence of clean faces was higher in the intervention villages than the control villages and this was statistically significant. Another trial compared eye washing to no treatment or to topical tetracycline alone or to a combination of eye washing and tetracycline drops in children with follicular trachoma. The trial found no statistically significant benefit of eye washing alone or in combination with tetracycline eye drops in reducing follicular trachoma amongst children with follicular trachoma.

\section{Authors' conclusions}

There is some evidence that face washing combined with topical tetracycline can be effective in reducing severe trachoma and in increasing the prevalence of clean faces. Current evidence does not however support a beneficial effect of face washing alone or in combination with topical tetracycline in reducing active trachoma.

\section{PLAINLANGUAGESUMMARY}

\section{Face washing promotion for reducing active trachoma}

Trachoma is an infectious eye disease. Active infection usually begins in childhood and is characterized by eye discharge, redness and irritation. Poor facial hygiene can lead to the disease spreading from person to person through eye-seeking flies or contaminated fingers. Face washing is promoted as part of the World Health Organization ' SAFE' strategy to eliminate blindness around the world. The review authors identified two randomized controlled trials with a total of 2560 participants set in Australia and Tanzania. One trial had face washing in combination with tetracycline as the intervention and tetracycline ointment alone as the control. The second trial compared eye washing to no treatment or to topical tetracycline alone or to a combination of eye washing and tetracycline drops in children with follicular trachoma. Both trials reported on active trachoma as an outcome measure but only one trial reported on severe trachoma and percentage of clean faces. The trials included in this review evaluated the effect of face washing over a three to 12 month period. There is some evidence that face washing combined with topical tetracycline can be effective in reducing severe trachoma and in increasing the prevalence of clean faces.

\section{B A C K G R O U N D}

\section{Epidemiology}

Trachoma is an infective eye disease caused by the microorganism Chlamydia trachomatis. Trachoma remains a major cause of avoidable blindness among underprivileged populations in many areas of Africa, Asia and the Middle East, where poverty, overcrowding, poor personal and environmental hygiene favor transmission of the disease. It is estimated that about 146 million people have active trachoma and nearly six million people are blind due to complications associated with repeat infections (WHO 1997a). The organism causing trachoma is spread from person to person by close contact in overcrowded living conditions, or through contaminated fingers or cloths used by mothers to wipe away dis- charges on the faces of children (ICEH 1999). Flies, which are attracted to eye and nasal secretions on the faces of infected children, are also believed to be risk factors in the transmission of the organism (ICEH 1999; West 1991).

\section{Presentation}

In communities where trachoma is endemic, infection usually begins in childhood and repeat episodes of infection cause distortion of the eyelids (entropion), in-turned eyelashes (trichiasis), corneal abrasion and ultimately blindness due to corneal opacity. Active trachoma is more commonly observed in children (Taylor 1985; West 1991). It is characterized by redness and discharge associated with inflammatory thickening of the upper tarsal conjunctiva (mucous membrane lining the inner surface of the upper eyelids) 
and follicles (whitish elevations within the conjunctiva). A simplified grading system for the assessment of trachoma and its complications in endemic communities has been published (Thylefors 1987) and discussed in a Cochrane review of antibiotics for trachoma (Evans 2011).

\section{The role of face washing in trachoma control}

Face washing is promoted by the World Health Organization (WHO) program for the global elimination of trachoma as part of the 'SAFE' strategy (WHO 1997b; WHO 1999). The SAFE strategy consists of surgery for trichiasis; antibiotics for infectious trachoma; facial cleanliness to reduce transmission; and environmental improvements (household sanitation and provision of clean water). The face washing component of this strategy aims to maintain clean faces in the community in order to reduce eyeseeking flies and person-to-person transmission of the trachoma organism. Face washing promotion as a community intervention can be combined with mass treatment with antibiotics in areas with high trachoma endemicity. Mass treatment with antibiotics aims to reduce the reservoir of Chlamydia trachomatis in the community while face washing aims to interrupt the cycle of infection and re-infection in the long term. The antibiotic and environmental arms of the SAFE strategy have been examined in other published Cochrane reviews (Evans 2011; Rabiu 2012).

\section{Rationale for a systematic review}

The face washing principle appears simple and theoretically sound, but whether this intervention can reduce transmission of trachoma in practice is now a focus of debate (Bailey 2001). Some narrative reviews of the literature have suggested that facial cleanliness may be useful in preventing trachoma (Emerson 2000; Pruss 2000). However, most of the data were obtained from observational studies and the methodological quality of the few controlled trials included was not reported. In this review we aim to summarize systematically, research evidence from trials of face washing promotion for preventing active trachoma in endemic communities. In communities where water is scarce, the uptake and practice of face washing may not be as good as in communities where water is freely available. The potential influence of water availability on outcomes will be considered in this review.

\section{O B JECT IVES}

The objective of this review was to assess the effects of face washing promotion on the prevalence of active trachoma in endemic communities.

\section{METHODS}

\section{Criteria for considering studies for this review}

\section{Types of studies}

We included randomized and quasi-randomized controlled trials.

\section{Types of participants}

Participants in the trials were people normally resident in communities where trachoma is endemic.

\section{Types of interventions}

We considered the following interventions:

1. face washing promotion versus no intervention;

2. face washing promotion plus mass antibiotic treatment versus mass antibiotic treatment alone.

Face washing promotion can be delivered by any means appropriate to the local setting such as: radio or television; health education leaflets; community leaders; religious gatherings; role-play; drama in village halls; school teachers; women groups; music etc. In trials where promotion of face washing was combined with mass antibiotic treatment, antibiotics considered include tetracycline ointment or capsules; azithromycin; or erythromycin, given at any dose or frequency.

\section{Types of outcome measures}

We considered the following outcomes.

1. Number of participants with active trachoma (TF or TI) at 6,12 , or greater than 12 months post-treatment allocation (age group as reported in trials).

Active trachoma was defined using the Thylefors 1987 scale. On this scale, active trachoma is categorized as TF or TI. TF is trachoma follicular inflammation and is defined as the presence of five or more follicles, each of which is at least $0.5 \mathrm{~mm}$ in diameter, on the flat surface of the upper tarsal conjunctiva. TI is trachoma intense inflammation and is defined as the presence of marked inflammatory thickening of the upper tarsal conjunctiva that obscures more than half of the deep conjunctival vessels.

We planned to include trials that used other trachoma grading scales to assess active trachoma, provided the scales used can be related to the Thylefors 1987 scale.

2. Number of participants with an unclean face at 6,12 , or greater than 12 months post treatment allocation (age group as reported in trials).

An unclean face was defined as the presence of eye or nasal discharge (WHO 2001) or any other definition used in trials.

3. Number of participants with severe trachoma.

Severe trachoma was not exclusively specified as an outcome in the protocol for this review. However, we felt it was important to

Face washing promotion for preventing active trachoma (Review) 
report it since one of the two trials that met the inclusion criteria defined and reported this outcome.

\section{Search methods for identification of studies}

\section{Electronic searches}

We searched the Cochrane Central Register of Controlled Trials (CENTRAL) 2011, Issue 8, part of The Cochrane Library. www.thecochranelibrary.com (accessed 2 September 2011), MEDLINE (January 1950 to September 2011), EMBASE (January 1980 to September 2011), Latin American and Caribbean Health Sciences Literature Database (LILACS) (January 1982 to September 2011), the metaRegister of Controlled Trials ( $m$ RCT) (www.controlled-trials.com) and ClinicalTrials.gov ( www.clinicaltrials.gov). There were no date or language restrictions in the electronic searches for trials. The electronic databases were last searched on 2 September 2011.

See: Appendices for details of search strategies for CENTRAL (Appendix 1), MEDLINE (Appendix 2), EMBASE (Appendix 3), LILACS (Appendix 4), $m$ RCT (Appendix 5) and ClinicalTrials.gov (Appendix 6).

\section{Searching other resources}

Trachoma experts that were contacted for potentially relevant studies include Hedley Peach and Sheila West. Denise Mabey was a peer reviewer and she provided information on potentially relevant studies. Existing reviews were identified and their citations were checked for relevant trials. We used the Science Citation Index to search for references that cite the studies that are included in the review.

\section{Data collection and analysis}

\section{Selection of studies}

Two review authors independently screened titles and abstracts found by the electronic searches. We retrieved for further assessment hard copies of trials that were potentially relevant to the review. Those that met the selection criteria were assessed for methodological quality. Disagreements were resolved by discussion.

\section{Assessment of methodological quality}

Two review authors independently assessed included trials using the following criteria based on Chapter 8 of the Cochrane Handbook for Systematic Reviews of Interventions (Higgins 2011):
1. Concealment of allocation: high, low, or unclear risk of bias. For trials with unclear concealment of allocation, further information was sought from primary investigators.

2. Baseline comparability between intervention and control groups with respect to prevalence of active trachoma: low risk of bias if no substantial differences present; unclear risk of bias if not reported or not known whether substantial differences exist; high risk of bias if substantial differences exist.

3. Comparability between intervention and control groups with respect to follow up: low risk of bias if no substantial differences in follow-up rates; unclear risk of bias if not reported or not known; high risk of bias if substantial differences exist in follow-up rates. 4. Intention-to-treat analysis: low risk of bias if performed; unclear risk of bias if not known; high risk of bias if not performed.

Masking of participants and providers were not used to assess trial quality in this review. The nature of the intervention made it difficult to successfully apply masking. Post hoc we decided to use masking of outcome assessors as a parameter of quality.

\section{Data collection}

Two review authors independently extracted data onto a standardized data extraction form. We compared extracted data and reconciled differences. Disagreements were resolved by a third review author. Where studies reported the outcomes in different ways, primary investigators were contacted for further information to allow transformation of data.

\section{Data analysis}

Only two trials met the inclusion criteria for this review and these used different interventions and methods for outcome assessment. A meta-analysis was considered inappropriate and a narrative summary of results is presented. If additional studies become available in the future we will use the following methods:

The specified outcomes are dichotomous therefore only relative risks will be calculated. Data will be combined in a meta-analysis if appropriate, using the random-effects model. If there are fewer than three studies and little evidence of heterogeneity a fixed-effect model will be used. In analyzing cluster-randomized trials, if we encounter trials where the units of allocation and analysis are different (i.e. the unit of allocation was the community and the unit of analysis was individuals in the community) and this has not been accounted for in the analysis, we will contact primary investigators for additional data to develop estimates of intracluster correlation coefficients or design effect to calculate more appropriate confidence intervals. If a meta-analysis is not possible, a tabulated summary of results will be presented.

We will not rely on statistical significance of a chi squared test to indicate heterogeneity but will consider this at all times during the review. The existence of heterogeneity may be apparent on visual examination of the forest plot. If present, heterogeneity will be explored using the following subgroups: 
1. Communities with available water supply versus communities with scarce water supply. Water availability is defined in this review as the presence of a functional water source within 30 minutes walk or a distance of less than four kilometers from all households within the community (WHO 2001) or any other definition used in the trials.

2. Communities with intense active trachoma versus communities with less intense active trachoma. Intense active trachoma is defined in this review as communities with a baseline prevalence of TF or TI equal to or greater than $20 \%$, while less intense is defined as communities with a prevalence of TF or TI less than 20\% (WHO 1997b).

If possible we will conduct a sensitivity analysis to investigate the influence of studies with quasi-random methods and those without concealment of allocation on the overall estimates of effect.

\section{RES U L T S}

\section{Description of studies}

See: Characteristics of included studies; Characteristics of excluded studies.

\section{Results of the search}

The electronic searches generated 67 citations and abstracts. These were screened and the full text of two potentially relevant articles were retrieved for further assessment. One of these met the criteria for inclusion (West 1995). The other was not a randomized controlled trial and therefore not included in the review (Sutter 1983). A trachoma research expert drew our attention to a randomized trial that was not published in a journal (Peach 1987). In all two randomized trials are included in the review.

Updated searches

An updated search done in October 2007 identified 66 new reports of trials. The Trials Search Co-ordinator scanned the search results and removed any references which were not relevant to the scope of the review. The full text of three articles were checked for potential inclusion, however all were excluded. Edwards 2006 and Rubinstein 2006 were reports of health education promotion of face washing and Khandekar 2006 treated face washing and environmental sanitation interventions as one outcome.

In September 2011 the electronic searches identified 91 additional references. One study underwent full-text assessment (King 2011) but was excluded as it evaluated a standardized definition of a clean face for trachoma prevention.

\section{Included studies}

See 'Characteristics of included studies' for further details.

\section{Setting and participants}

This review includes data from a total of 2560 participants in two trials. West 1995 was undertaken in Kongwa, Tanzania. In this trial a total of 1417 children, aged one to seven years from six villages, were randomized in three pairs to intervention or control. Peach 1987 was undertaken in the Northern Territory of Australia. In this trial 36 aboriginal communities were randomized to one of three intervention arms or one control arm. A total of 2530 children aged five to 14 were screened for follicular trachoma. A few more children above the age of 14 and some of preschool age were also screened. Of the total number of children screened in the participating communities, only 1143 children with follicular trachoma were recruited into the trial.

\section{Interventions}

In West 1995, 680 children from three villages were randomized to face washing promotion combined with tetracycline and 737 children from three villages were randomized to tetracycline ointment alone. Face washing promotion was community based and consisted of neighborhood meetings to build consensus for increasing face washing and reinforcement activities such as school plays, seminars with the traditional healers and meetings with other village groups. Face washing promotion was carried out for one month during and after mass treatment with tetracycline. Tetracycline ointment was administered topically once daily for 30 days. In Peach 1987, 374 were randomized to tetracycline eye drops, 246 children were randomized to eye washing, 312 children were randomized to eye washing combined with tetracycline eye drops, and 211 children were randomized to the no treatment group. Children in the eye washing group had their eyes washed daily by school teachers for three months. Those in the tetracycline group had tetracycline eye drops applied daily for one week every month for three months. For the purpose of this review, data for the comparison between eye washing versus no treatment, and eye washing combined with eye drops versus eye drops alone are reported.

\section{Outcome measures}

In West 1995, outcomes reported include active trachoma, severe trachoma and clean faces. Trachoma was graded using the Thylefors 1987 scale. Severe trachoma was defined exclusively in the trial as 15 or more follicles, or the presence of inflammation that obscured all vessels of the tarsal plate. We extrapolated and extracted data from graphs presented in the report of the trial, as raw data were unavailable. These extrapolated data should be regarded as best approximations to the true figures. Our protocol specified 'unclean faces' as the outcome of interest, but this was reported as 'clean faces' in this trial. We have elected to present the outcome as reported in the trial as it would be difficult to transform the data without sufficient information from the trialists.

Face washing promotion for preventing active trachoma (Review) 
In Peach 1987, outcome was reported as the proportion of children with follicular trachoma who had follicles at three months after the intervention. The Aboriginal Health Workers simplified grading scheme was used to assess the presence of follicles as indicating active trachoma. Although this scale can be crudely compared to the TF grading on the Thylefors scale, it may have a lower specificity because of the tendency to classify participants with fewer than five follicles as having active trachoma.

\section{Excluded studies}

See Characteristics of excluded studies for further details.

\section{Risk of bias in included studies}

In West 1995 there was no information on how randomization was completed and whether allocation of villages to intervention or control was concealed. Baseline prevalences in active trachoma between comparison villages were not substantially different. Although $92 \%$ of the enrolled participants were followed up for one year, information regarding similarity of follow-up rates between comparison groups was not provided in the report. Information on whether analysis of results was based on an intention to treat principle was not provided in the report. We note that this trial masked outcome assessment by taking photographs of tarsal plate read by an examiner who was not aware of the randomization status of the villages.

In Peach 1987, details of how randomization was completed and concealment of allocation were not available in the report. Additional information from the author reveals that a random number table was used to allocate communities to the interventions or control group. The allocation was done after the initial screening by someone who was unaware of the prevalence of trachoma and unfamiliar with the communities, including their school teachers and health workers. It is unclear whether baseline prevalence of trachoma was similar among the comparison groups. Information on the number of communities randomized to each experimental group was not available in the report. However, during further correspondence the authors suggest that about nine communities were randomized to each arm. Almost $89 \%$ of enrolled participants were followed up for three months. All participants lost to follow up were assumed to have follicles at the end of the study and the intention to treat principle was applied in the analysis of results. Outcome was assessed by trachoma workers who were unaware of treatment allocation to the communities. Steps were taken to ensure that the outcome assessors did not learn which groups the communities were allocated to.

Table 1 gives the results of the assessment of methodological quality of the included trials.

\section{Effects of interventions}

The two trials included were different in several respects, particularly with regard to types of intervention and definition of outcome measures. Therefore a meta-analysis was not considered appropriate. A narrative summary of the results is presented.

\section{Active trachoma (follicular or TF or TI)}

In West 1995, face washing combined with antibiotics was compared to antibiotics alone in three pairs of villages. In pair one, the percentage prevalence of active trachoma was lower in the village that received a combination of face washing and antibiotics than the village that received antibiotics alone at 12 months follow up (approximately 55\% compared to $60 \%$ ). In a second pair of villages, the percentage of active trachoma was also lower in the combination village than the antibiotic alone village (approximately $40 \%$ compared to $50 \%$ ). However in a third pair of villages, the percentage of active trachoma in the combination village was higher than the antibiotics alone village (approximately $70 \%$ compared to $65 \%$ ). The overall results for all the combination villages compared to the antibiotics alone villages suggest a reduction in the odds of any trachoma but this effect was not statistically significant (odds ratio (OR) $0.81,95 \%$ confidence interval (CI) 0.42 to 1.59$)$.

In Peach 1987, 191/246 children (77.6\%) in the eye washing arm had follicles at three months compared to $160 / 211$ (75.8\%) in the no treatment arm. The difference was not statistically significant $(\mathrm{P}=0.73)$. In the eye washing/eye drop combination arm, $215 / 312(68.9 \%)$ had follicles at three months compared to $250 /$ $374(66.8 \%)$ in the eye drop only arm. The difference was not statistically significant $(P=0.62)$. When a logit model was fitted to the data, taking age of participants, geographical location and trachoma outcome assessors into account, the results show that the odds of having follicular trachoma was higher in the eye drop only arm compared to the eye washing-eye drop combination (odds 1.17 to 1.00 ) but these odds were not significantly greater than 1). The odds of having follicular trachoma in the no treatment group compared to the eye washing group were similar (odds 1.02 to 1.00$)$.

\section{Severe trachoma}

In West 1995 the three pairs of villages were also compared with respect to prevalence of severe trachoma. In pair one, the percentage prevalence of severe trachoma was lower in the village that received a combination of face washing and antibiotics than the village that received antibiotics alone at 12 months follow up (approximately $8 \%$ compared to $14 \%$ ). In a second pair of villages, the percentage of active trachoma was also lower in the combination village than the antibiotic alone village (approximately $6 \%$ compared to $14 \%)$. However in a third pair of villages, the percentage of active trachoma in the combination village was slightly higher than the

Face washing promotion for preventing active trachoma (Review) 
antibiotics alone village (approximately $10 \%$ compared to $8 \%$ ). The overall results after adjustments for age and baseline trachoma status suggests a reduction in the odds of severe trachoma by the face washing antibiotic combination compared to antibiotic alone and this effect was statistically significant (OR 0.62, 95\% CI 0.40 to 0.97 ). At six months follow up, there were no differences in the prevalence of severe trachoma between the intervention and control groups in the three pairs of villages.

Peach 1987 did not report this outcome.

\section{Clean faces}

In West 1995 the percentage of children with clean faces was consistently higher in the face washing-antibiotic combination villages than the antibiotic alone villages. Total results showed an increase in the percentage of children with clean faces in the face washing/antibiotic combination villages from $18 \%$ at baseline to $33 \%$ at six months and $35 \%$ at 12 months follow up. There was a smaller increase in the percentage of children with clean faces in the antibiotic alone group (from $19 \%$ at baseline to $30 \%$ at six months and $26 \%$ at 12 months). The difference in the proportion of children with clean faces in the intervention villages compared to the control villages was statistically significant $(\mathrm{P}<0.05)$.

Peach 1987 did not report this outcome.

\section{DISCUSSION}

Although two trials are included in this review, a meta-analysis was not performed. This was because of notable clinical heterogeneity between the two trials, particularly with regard to intervention strategies and outcome definition. Although the report of the design and conduct of both trials suggests notable efforts by the investigators to strengthen the quality, lack of adequate information made it impossible objectively to assess the trials against some key quality parameters specified in the review (see section on methodological quality and additional Table 1). Outcomes were reported at three months in Peach 1987. Although the follow-up period fell short of what was specified in our protocol, we did not exclude the data from this trial in view of the paucity of randomized trials.

\section{Active trachoma}

It is unclear why face washing promotion combined with tetracycline had an effect in reducing active trachoma in two pairs of villages but no effect in a third pair in West 1995. Differences in baseline characteristics such as prevalence of trachoma, intensity of transmission, availability or access to water supplies between the third pair and the first two pair of villages may be important in explaining the differences in benefit. However, the overall results for the face washing/tetracycline combination villages compared to the tetracycline only villages suggest a modest beneficial effect of face washing in reducing active trachoma at 12 months, although this was not statistically significant.

Peach 1987 suggests no benefit for face washing compared with no treatment. The raw data also show no benefit for the face washing/ eye drops combination in comparison to eye drops alone. The age of participants varied and the authors observed a higher proportion of severe trachoma among older children. There were variations in the prevalence of trachoma in the different geographical locations from which the participating communities were drawn as well as slight differences in the diagnostic competence of outcome assessors. The authors hypothesised that community randomization as done in the trial may not have adequately controlled for these factors hence the need to account for them in the logit model. After fitting the data to a logit model to control for perceived imbalances in the ages of participants, geographical location and outcome assessors, a marginal but not statistically significant benefit is suggested for the face washing/eye drops combination over the eye drops alone group. The report however does not state whether the analysis of results in a logit model was planned in advance or simply informed by the apparent lack of effect suggested by the raw data.

The lack of effect of face washing in Peach 1987 can be explained by a number of factors. Firstly, the trachoma grading system used in the trial can potentially influence the results. Participants were recruited into the trial on the basis of whether follicles or papillae were present. Based on this definition, participants with follicles/ papillae from causes other than active trachoma could have been included. For this group of people, treatment would appear to have no benefit if what is being treated is not trachoma. Furthermore, if participants had trachoma which was not intense, the effect of the face washing may not be readily apparent. Secondly, in analyzing the results using the intention to treat principle, the authors assumed that the participants lost to follow up had follicular trachoma at the end of the study. If this assumption was inaccurate and there were more participants lost to follow up in a treatment group compared to control, as was the case with the eye washing arm (17\% versus $10.4 \%)$, treatment might appear to be ineffective compared to control. However, a sensitivity analysis with the missing participants excluded from analysis did not alter the results. Thirdly, the intervention was administered for only three months. A longer intervention period and follow up may have significantly altered the results. Fourthly, in Peach 1987, face washing was applied to children with already established disease rather than the whole population at risk, and outcome was measured in this group of children. The face washing strategy aims to reduce active trachoma in endemic communities mainly by reducing the transmission of the disease. A better measure of effect would have been to evaluate the magnitude of the disease amongst the whole population or subset of the population rather than amongst persons with the disease, or to determine the number of new cases of disease since institution of the intervention. It is also unclear 
how much impact on transmission can be achieved by applying face washing only to individuals with the disease in endemic communities. The true impact of face washing on active trachoma in the communities might be better evaluated by a study design in which face washing is applied to whole populations at risk rather than only those with the disease.

\section{Severe trachoma}

As for active trachoma, benefits of face washing in reducing the prevalence of severe trachoma were apparent in the first and second pairs of villages in West 1995 at 12 months follow up. In the third pair, there appeared to be no benefit. The overall results for all the villages after adjusting for age and baseline trachoma status showed a benefit of face washing in reducing severe trachoma in the intervention villages compared to the control villages at 12 months follow up. It is probable that participants with severe active trachoma represent a subgroup with more intense transmission and therefore face washing, which aims to break transmission, would be more likely to show a stronger effect within this subgroup. On the other hand, the appropriateness of combining the results from the three pairs of villages is questionable, since presumably the villages were paired because of some differences between them. It is unclear why face washing showed no comparative benefit in the three pairs of villages at six months follow up. Apparent benefit at 12 months underscores the importance of a longer follow-up period to demonstrate impact of the intervention.

\section{Clean faces}

We note with interest that the percentage of participants with clean faces increased in both intervention and control groups over 12 months, even though the increase was higher in the intervention group. However, a statistically significant difference in the percentage of clean faces between the intervention and control groups at 12 months suggests a benefit of face washing promotion.

Previous narrative reviews of the literature have reported possible beneficial effects of face washing in preventing active trachoma. The conclusions of these narrative reviews were based on data obtained from one trial and a number of observational studies. This review assessed the methodological quality of the trials included and found that some important quality parameters were not adequately addressed.

\section{A U THORS' CONCLUSIONS}

\section{Implications for practice}

Evidence from one trial suggests that face washing can be effective in increasing facial cleanliness and in reducing severe trachoma, but its effect in reducing active trachoma is inconclusive. In another trial, there was no evidence of effect of face washing alone or in combination with tetracycline in reducing active trachoma in children with already established disease.

\section{Implications for research}

The trials included in this review evaluated the effect of face washing over a three to 12 month period. However, it is unclear whether this time period is long enough for a face washing promotional activity to demonstrate impact of the intervention. Therefore, future research should include longer follow-up periods and also address the questions of whether reinforcement activities are required over time to improve outcome. The reporting of the methodology of trials should be complete to enable reviewers and readers to assess the validity of their conclusions.

\section{ACKNOWLEDGEMENTS}

We are grateful to the editorial team of the Cochrane Eyes and Vision Group for executing the electronic searches. We also gratefully acknowledge the tremendous advice and co-operation we received from Professor Peach, the author for correspondence of one of the trials included in this review. We acknowledge the invaluable contribution of Denise Mabey who peer reviewed this review and also of Catey Bunce, Marie Diener-West and Roberta Scherer for their statistical and methodological advice.

\section{REFER E N C E S}

\section{References to studies included in this review}

Peach 1987 \{published data only\}

Peach H, Piper S, Devanesen D, Dixon B, Jefferies C, Braun P, et al.Northern Territory Trachoma Control and Eye Health Committee's Randomised Controlled Trial of the Effect of Eye Drops and Eye Washing on Follicular Trachoma Among Aboriginal Children. Report of the Northern Territory Trachoma Control and Eye Health
Committee Incorporated 1987:1-33.

West 1995 \{published data only\}

West S, Munoz B, Lynch M, Kayongoya A, Chilangwa Z, Mmbaga BBO, et al.Impact of face-washing on trachoma in Kongwa, Tanzania. Lancet 1995;345(8943):155-8.

\section{References to studies excluded from this review}


Edwards 2006 \{published data only\}

Edwards T, Cumberland P, Hailu G, Todd J. Impact of health education on active trachoma in hyperendemic rural communities in Ethiopia. Ophthalmology 2006;113(4): $548-55$.

Khandekar 2006 \{published data only\}

Khandekar R, Ton TK, Do Thi P. Impact of face washing and environmental improvement on reduction of active trachoma in Vietnam-a public health intervention study. Ophthalmic Epidemiology 2006;13(1):43-52.

King 2011 \{published data only\}

King JD, Ngondi J, Kasten J, Diallo MO, Zhu M, Cromwell EA, et al.Randomised trial of face-washing to develop a standard definition of a clean face for monitoring trachoma control programmes. Transactions of the Royal Society of Tropical Medicine and Hygiene 2011;105:7-16.

Rubinstein 2006 \{published data only\} Rubinstein RA, Lane SD, Sallam SA, Sheta AS, Gad ZM, Sherif AR, et al.Controlling blinding trachoma in the Egyptian Delta: Integrating clinical, epidemiological and anthropological understandings. Anthropology \& Medicine 2006;13(2):99-118.

Sutter 1983 \{published data only\}

Sutter E, Ballard R. Community participation in the control of trachoma in Gazankulu. Social Science and Medicine 1983;17(22):1813-7.

\section{Additional references}

\section{Bailey 2001}

Bailey R, Lietman T. The SAFE strategy for the elimination of trachoma by 2020: will it work?. Bulletin of the World Health Organization 2001;79(3):233-6.

Emerson 2000

Emerson PM, Cairncross S, Bailey RL, Mabey DCW. Review of the evidence base for the 'F' and 'E' components of the SAFE strategy for trachoma control. Tropical Medicine and International Health 2000;5(8):515-27.

Evans 2011

Evans JR, Solomon AW. Antibiotics for trachoma. Cochrane Database of Systematic Reviews 2011, Issue 3. [DOI: 10.1002/14651858.CD001860.pub3]

Glanville 2006

Glanville JM, Lefebvre C, Miles JN, Camosso-Stefinovic J. How to identify randomized controlled trials in MEDLINE: ten years on. Journal of the Medical Library Association 2006; 94(2):130-6.

\section{Higgins 2011}

Higgins JPT, Altman DG, Sterne JAC (editors). Chapter 8: Assessing risk of bias in included studies. In: Higgins JPT, Green S (editors). Cochrane Handbook for Systematic Reviews of Interventions Version 5.1.0 (updated March 2011). The Cochrane Collaboration, 2011. Available from www.cochrane-handbook.org.

\section{ICEH 1999}

International Centre for Eye Health. Slides/Text Teaching Series: No. 7 Trachoma. Second Edition. London: International Centre for Eye Health, 1999.

Pruss 2000

Pruss A, Mariotti SP. Preventing trachoma through environmental sanitation: a review of the evidence base. Bulletin of the World Health Organization 2000;78(2): 258-66.

Rabiu 2012

Rabiu M, Alhassan M, Ejere H, Evans JR. Environmental sanitary interventions for preventing active trachoma. Cochrane Database of Systematic Reviews 2012, Issue 2. [DOI: 10.1002/14651858.CD004003.pub4]

Taylor 1985

Taylor HR, Velasco FM, Sommer A. The ecology of trachoma: an epidemiological study in southern Mexico. Bulletin of the World Health Organization 1985;63(3): 559-67.

Thylefors 1987

Thylefors B, Dawson CR, Jones BR, West SK, Taylor HR. A simple system for the assessment of trachoma and its complications. Bulletin of the World Health Organization 1987;65(4):477-83.

West 1991

West SK, Munoz B, Turner VM. The epidemiology of trachoma in central Tanzania. International Journal of Epidemiology 1991;20(4):1088-92.

WHO 1997a

World Health Organization. Blindness and Visual Disability. Part II of VII: Major Causes Worldwide. Fact sheet N 143. www.who.int/inf-fs/en/fact143.html 1997 (accessed 13 September 2001).

\section{WHO 1997b}

World Health Organization. Future approaches to trachoma control, report of a global scientific meeting. WHO//PBL/ 96.561997.

\section{WHO 1999}

World Health Organization. Report of the third Meeting of the WHO Alliance for the Global Elimination of Trachoma. WHO/PBL/GET/99.3 www.who.int/pbd/trachoma/ Reports/reports.htm 1999 (accessed 16 February 2002).

\section{WHO 2001}

Negrel AD, Taylor HR, West S. Guidelines for the Rapid Assessment for Blinding Trachoma. WHO/PBD/GET/ 00.82001 .

\section{References to other published versions of this review}

Ejere 2004

Ejere HOD, Alhassan MB, Rabiu M. Face washing promotion for preventing active trachoma. Cochrane Database of Systematic Reviews 2004, Issue 3. [DOI: 10.1002/14651858.CD003659.pub2]

* Indicates the major publication for the study

Face washing promotion for preventing active trachoma (Review) 


\section{CHARACTERISTICS OF STUDIES}

\section{Characteristics of included studies [ordered by study ID]}

\section{Peach 1987}

\begin{tabular}{|c|c|c|}
\hline Methods & \multicolumn{2}{|c|}{$\begin{array}{l}36 \text { aboriginal communities were randomized in stages to four experimental groups after } \\
\text { stratification by geographical location } \\
\text { Method of randomization: not stated } \\
\text { Unit of randomization: communities, but individuals where analyzed } \\
\text { Masking: outcome assessors masked but method of masking unclear } \\
\text { Analysis was by intention-to-treat principle (participants lost to follow up were assumed to } \\
\text { have follicles at the end of the study) }\end{array}$} \\
\hline Participants & \multicolumn{2}{|c|}{$\begin{array}{l}\text { Country: Northern Territory of Australia } \\
\text { Participants: children aged five years and above drawn from } 36 \text { aboriginal communities } \\
\text { Age range: most participants were between } 5 \text { and } 14 \text { years, although a small proportion of } \\
\text { children were older than } 14 \text { and a small proportion were pre-school } \\
\text { Total number of children randomized: } 1143\end{array}$} \\
\hline Interventions & \multicolumn{2}{|c|}{$\begin{array}{l}\text { Treatment: } \\
\text { 1. Tetracycline eye drops daily for one week every month for } 3 \text { months ( } 374 \text { children } \\
\text { randomized) } \\
\text { 2. Eye washing daily for } 3 \text { months ( } 246 \text { children randomized) } \\
\text { 3. Tetracycline eye drops plus eye washing ( } 312 \text { children randomized) } \\
\text { Control: } \\
\text { No treatment ( } 211 \text { children) }\end{array}$} \\
\hline Outcomes & \multicolumn{2}{|c|}{ Follicular trachoma (proportion of children with follicular trachoma at 3 months) } \\
\hline \multicolumn{3}{|l|}{ Notes } \\
\hline \multicolumn{3}{|l|}{ Risk of bias } \\
\hline Bias & Authors' judgement & Support for judgement \\
\hline Allocation concealment (selection bias) & Unclear risk & Unclear \\
\hline
\end{tabular}

\section{West 1995}

Methods

Six villages were randomized in three pairs to intervention or control Method of randomization: unclear

Masking: outcome assessors masked. Assessors examined photographs of tarsal plates for follicles

Participants

Country: Kongwa, Tanzania

Participants: Children aged 1 to 7 years drawn from 6 trachoma endemic villages

Total number of children randomized: 1417 
West 1995 (Continued)

\begin{tabular}{|c|c|c|}
\hline Interventions & \multicolumn{2}{|c|}{$\begin{array}{l}\text { Treatment: } \\
\text { Face washing promotion combined with mass tetracycline ointment ( } 680 \text { children) } \\
\text { Control: } \\
\text { Mass tetracycline ointment only ( } 737 \text { children). Tetracycline ointment was administered } \\
\text { topically once daily for } 30 \text { days }\end{array}$} \\
\hline Outcomes & \multicolumn{2}{|l|}{$\begin{array}{l}\text { 1. Active trachoma } \\
\text { 2. Severe trachoma } \\
\text { 3. Clean face }\end{array}$} \\
\hline \multicolumn{3}{|l|}{ Notes } \\
\hline \multicolumn{3}{|l|}{ Risk of bias } \\
\hline Bias & Authors' judgement & Support for judgement \\
\hline Allocation concealment (selection bias) & Unclear risk & Unclear \\
\hline
\end{tabular}

Characteristics of excluded studies [ordered by study ID]

\begin{tabular}{ll}
\hline Study & Reason for exclusion \\
\hline Edwards 2006 & Study intervention is health education promotion of face washing \\
\hline Khandekar 2006 & $\begin{array}{l}\text { Unable to separate the effect of face washing from environmental sanitation interventions as both were indirectly } \\
\text { examined as "one intervention" }\end{array}$ \\
\hline King 2011 & Study aim was to develop standardized definition for a clean face in trachoma prevention \\
\hline Rubinstein 2006 & Study intervention is health education promotion of face washing \\
\hline Sutter 1983 & Not a randomized controlled trial \\
\hline
\end{tabular}




\section{DATA ANDANALYSES}

This review has no analyses.

\section{ADDITIONAL TABLES}

Table 1. Quality assessment

\begin{tabular}{lllllll}
\hline Trial ID & $\begin{array}{l}\text { Method randomiza- } \\
\text { tion }\end{array}$ & Allocation conceal. & Baseline compar. & Attrition & Intention-to-treat \\
\hline Peach 1987 & Unclear risk & Unclear risk & Low risk & Unclear risk & Unclear risk \\
\hline West 1995 & Low risk & ? Low risk & Unclear risk & Low risk & Low risk \\
\hline
\end{tabular}

\section{A P P E N D I C E S}

\section{Appendix I. CENTRAL search strategy}

\#1 MeSH descriptor Trachoma

\#2 MeSH descriptor Chlamydia trachomatis

\#3 (trachom*)

\#4 (tracom*)

\#5 (follicular near conjunctivitis)

\#6 (intense near conjunctivitis)

\#7 (\#1 OR \#2 OR \#3 OR \#4 OR \#5 OR \#6)

\#8 MeSH descriptor Hygiene

\#9 MeSH descriptor Face

\#10 face-wash or facewash*

\#11 face near wash*

\#12 facial near wash*

\#13 face near clean*

\#14 facial near clean*

\#15 face near hygien*

\#16 facial near hygien*

\#17 (\#8 OR \#9 OR \#10 OR \#11 OR \#12 OR \#13 OR \#14 OR \#15 OR \#16)

\#18 (\#7 AND \#17) 


\section{Appendix 2. MEDLINE (OVID) search strategy}

1 randomized controlled trial.pt.

2 (randomized or randomised).ab,ti.

3 placebo.ab,ti.

4 dt.fs.

5 randomly.ab,ti.

6 trial.ab,ti.

7 groups.ab,ti.

8 or/1-7

9 exp animals/

10 exp humans/

119 not (9 and 10)

128 not 11

13 exp trachoma/

14 exp chlamydia-trachomatis/

15 or/13-14

16 trac?oma\$.tw.

17 (follicular adj3 conjunctivitis).tw.

18 (intense adj3 conjunctivitis).tw.

19 or/16-18

2015 or 19

21 exp hygiene/

22 exp facel

23 or $/ 21-22$

24 ((face\$ adj3 wash\$) or clean\$ or hygien\$).tw.

25 ((facial adj3 wash\$) or clean\$ or hygien\$).tw.

26 (face adj1 wash\$).tw.

27 or/ $24-26$

2823 or 27

2920 and 28

3012 not 29

3112 and 29

The search filter for trials at the beginning of the strategy is from the published paper by Glanville et al (Glanville 2006).

\section{Appendix 3. EMBASE (OVID) search strategy}

1 exp randomized controlled trial/

2 exp randomization/

3 exp double blind procedure/

4 exp single blind procedure/

5 random $\$$.tw.

6 or/ $1-5$

7 (animal or animal experiment).sh.

8 human.sh.

97 and 8

107 not 9

116 not 10

12 exp clinical trial/

13 (clin\$ adj3 trial\$).tw.

14 ((singl\$ or doubl\$ or trebl\$ or tripl\$) adj3 (blind\$ or mask\$)).tw.

15 exp placebo/

16 placebo\$.tw.

Face washing promotion for preventing active trachoma (Review)

Copyright $\odot 2012$ The Cochrane Collaboration. Published by John Wiley \& Sons, Ltd. 
17 random\$.tw.

18 exp experimental design/

19 exp crossover procedure/

20 exp control group/

21 exp latin square design/

22 or/12-21

2322 not 10

2423 not 11

25 exp comparative study/

26 exp evaluation/

27 exp prospective study/

28 (control\$ or prospectiv\$ or volunteer\$).tw.

29 or $/ 25-28$

3029 not 10

3130 not (11 or 23$)$

3211 or 24 or 31 )

33 exp trachoma/

34 exp chlamydia-trachomatis/

35 or/33-34

36 trac?oma $\$$.tw.

37 (follicular adj3 conjunctivitis).tw.

38 (intense adj3 conjunctivitis).tw.

39 or/36-38

4035 or 39

41 exp personal hygiene/

42 exp facel

43 or/ $41-42$

44 ( (face\$ adj3 wash\$) or clean\$ or hygien\$).tw.

45 ((facial adj3 wash\$) or clean\$ or hygien\$).tw.

46 (face adj1 wash\$).tw.

47 or $/ 44-46$

4843 or 47

4940 and 48

5032 and 49

\section{Appendix 4. LILACS search strategy}

trachoma $\$$ and face or facial\$ and wash\$ or clean\$ or hygien\$

Appendix 5. metaRegister of Controlled Trials search strategy

(wash or clean or hygiene) and trachoma 


\section{Appendix 6. ClinicalTrials.gov search strategy}

(Wash OR Clean OR Hygiene) AND Trachoma

\section{WHAT'S NEW}

Last assessed as up-to-date: 2 September 2011.

\begin{tabular}{l|l|l}
\hline Date & Event & Description \\
\hline 3 February 2012 & New citation required but conclusions have not changed & $\begin{array}{l}\text { Issue 4, 2012: Risk of bias assessment has been amended } \\
\text { to reflect updated changes by The Cochrane Collabo- } \\
\text { ration }\end{array}$ \\
\hline 3 February 2012 & New search has been performed & $\begin{array}{l}\text { Issue 4, 2012: Updated searches yielded no new studies } \\
\text { for inclusion }\end{array}$ \\
\hline
\end{tabular}

\section{H I S T O R Y}

Protocol first published: Issue 2, 2002

Review first published: Issue 3, 2004

\begin{tabular}{l|ll}
\hline Date & Event & Description \\
\hline 23 October 2008 & Amended & Converted to new review format. \\
\hline 20 February 2008 & New search has been performed & $\begin{array}{l}\text { Issue 2 2008: three new trials were identified in an up- } \\
\text { dated search but were excluded }\end{array}$ \\
\hline 31 March 2004 & New citation required and conclusions have changed & Substantive amendment. \\
\hline
\end{tabular}

\section{CONTRIBUTIONSOFAUTHORS}

Conceiving the review: $\mathrm{HE}$

Coordinating the review: $\mathrm{HE}$

Data collection for the review: HE

Screening search results: HE, MA

Organising retrieval of papers: $\mathrm{HE}$

Screening retrieved papers against inclusion criteria: HE, MA, MR

Appraising quality of papers: HE, MA, MR

Abstracting data from papers: HE, MA, MR

Writing to authors of papers for additional information: HE

Face washing promotion for preventing active trachoma (Review)

Copyright $\odot 2012$ The Cochrane Collaboration. Published by John Wiley \& Sons, Ltd. 
Resolving disagreements between reviewers: MR

Entering data into RevMan: HE

Interpretation of the data: $\mathrm{HE}, \mathrm{MR}$

Writing of the review: HE, MA, MR

Updating the review: HE, Cochrane Eyes and Vision Group US Satellite (Michael Marrone)

\section{DECLARATIONSOF INTEREST}

None known.

\section{SOURCES OF SUPPORT}

\section{Internal sources}

- No sources of support supplied

\section{External sources}

- Effective Health Care Alliance Project, International Health Division, Liverpool School of Tropical Medicine, UK.

- National Eye Centre, Nigeria.

\section{NDEX TERMS}

\section{Medical Subject Headings (MeSH)}

*Chlamydia trachomatis; *Face; Anti-Bacterial Agents [administration \& dosage]; Randomized Controlled Trials as Topic; Skin Care [ ${ }^{*}$ methods]; Tetracycline [administration \& dosage]; Trachoma [* prevention \& control]

\section{MeSH check words}

Humans 